inductors, and pads in silicon process, Ph.D. Thesis, National Central University, Taiwan, 2004.

6. Y.S. Lin and S.S. Lu, An analysis of the anomalous dip in scattering parameter $\mathrm{S}_{11}$ of $\mathrm{InGaP} / \mathrm{GaAs}$ heterojunction bipolar transistors (HBTs), Jpn J Appl Phys B 43 (2004), L803-L805.

7. Y.S. Lin and S.S. Lu, An analysis of small-signal gate-drain resistance effect on RF power MOSFETs, IEEE Trans Electron Devices 50 (2003), 525-528.

8. H.Y. Tu, Y.S. Lin, P.Y. Chen, and S.S. Lu, An analysis of the anomalous dip in scattering parameter $\mathrm{S}_{22}$ of $\mathrm{InGaP} / \mathrm{GaAs}$ heterojunction bipolar transistors (HBTs), IEEE Trans Electron Devices 49 (2002), 1831-1833.

9. S.S. Lu, C.C. Meng, T.W. Chen, and H.C. Chen, The origin of the kink phenomenon of transistor scattering parameter $\mathrm{S}_{22}$, IEEE Trans Microwave Theory Tech 49 (2001), 333-340.

(C) 2007 Wiley Periodicals, Inc.

\section{A CLOSED-FORM SOLUTION TO THE ASYMPTOTIC PART OF THE MOM IMPEDANCE MATRIX AND THE MOM EXCITATION VECTOR FOR PRINTED STRUCTURES ON PLANAR GROUNDED DIELECTRIC SLABS}

\author{
O. Bakýr and V. B. Ertürk \\ Department of Electrical and Electronics Engineering, Bilkent \\ University, TR-06800, Bilkent, Ankara, Turkey
}

\section{Received 18 August 2006}

ABSTRACT: In the spectral domain method of moments (MoM) solution of printed structures on planar grounded dielectric slabs, the infinite double integrals which appear in the asymptotic parts of the MoM impedance matrix and the MoM excitation vector elements, have been previously transformed to one-dimensional finite integrals, which have been numerically computed using the highly specialized "International Mathematics and Statistics Library” subroutines. In this paper, these one-dimensional integrals are evaluated in closed-form, resulting in an improved efficiency and accuracy for the rigorous investigation of printed antennas and complex millimeter and microwave integrated circuits. Numerical results in the form of mutual impedance between two expansion functions and input impedance of various microstrip antennas are presented to assess the accuracy of these closed-form expressions. (C) 2007 Wiley Periodicals, Inc. Microwave Opt Technol Lett 49: 882-886, 2007; Published online in Wiley InterScience (www. interscience.wiley.com). DOI 10.1002/mop.22274

Key words: method of moments; microstrip antennas; (M)MIC structures; spectral domain analysis

\section{INTRODUCTION}

The spectral domain Method of Moments (MoM) solution to the integral equation is one of the widely used techniques for the analysis and design of printed antennas and arrays as well as microwave- and millimeter-wave integrated circuits due to its high accuracy and relatively good modeling capabilities [1-7]. In this technique, the elements in the MoM matrix equation are expressed as integrals over a spectrum of wave functions, rather than as integrals over the physical space of the expansion and weighting functions. Therefore, the impedance-matrix (and the MoM excitation vector in the case of probe-fed structures) elements are expressed in terms of double integrals with limits extended to infinity, which must be evaluated numerically. Unfortunately, the slowly convergent and highly oscillating behaviors of the inte- grands make filling the impedance-matrix elements as the most time-consuming part in the MoM solution, in particular for electrically large structures. Besides, such behaviors may create accuracy problems as well. Thus, various techniques have been developed related to the spectral domain evaluation of the impedance matrix and the excitation vector entries [2-7]. Among them, in Refs. 5 and 6, the authors have successfully derived an analytical technique for the fast and accurate evaluation of the asymptotic part of the impedance matrix when triangular edge mode and roof-top subdomain basis functions are used in the spectral domain MoM solution for printed narrow strips and antennas. Basically, they provide an analytical transformation from an infinite double (2D) integral to a finite one-dimensional (1D) integral for the asymptotic part of the impedance matrix, thereby reducing the CPU time markedly and improving the accuracy regardless of the lateral separation between the basis and testing functions. Recently, the same method has been applied to the MoM excitation vector for probe-fed planar microstrip antennas [7].

In all these three studies [5-7], the resulting one-dimensional finite integrals are computed using the "International Mathematics and Statistics Library (IMSL)" subroutines DQDAGP (if there is a singularity) or DQDAGS, which are high-quality adaptive integral routines. Unfortunately, these routines are highly specialized and may not be available on all platforms. Moreover, using standard numerical integration techniques instead of these IMSL routines may yield accuracy problems. Therefore, in this paper we provide closed-form results for these 1D integrals. Consequently, the asymptotic parts of both the impedance matrix and the excitation vector are evaluated completely in closed-form, which results in a further reduction in the CPU time and a further improvement in the accuracy for the evaluation of the MoM matrix and the excitation vector entries. Besides, these closed-form expressions eliminate the need for such highly specialized subroutines for such problems.

In Section 2, closed-form evaluation of the aforementioned 1D integrals is presented. In Section 3, numerical results involving the mutual impedance between two expansion functions and the input impedance of various microstrip antennas are calculated and compared with the published results to assess the accuracy of these closed-form expressions. An $e^{j w t}$ time dependence is assumed and suppressed throughout this paper.

\section{FORMULATION}

In the spectral domain MoM solution of printed structures on planar grounded dielectric slabs, using Galerkin's method and employing the asymptotic extraction technique, the impedance matrix elements can be expressed in the form of

$$
\begin{aligned}
Z_{m^{\prime} n^{\prime} m n}^{p q}= & -\frac{1}{4 \pi^{2}} \int_{\infty}^{\infty} \int_{\infty}^{\infty} \tilde{J}_{m^{\prime} n^{\prime}}^{p^{*}}\left(k_{x}, k_{y}\right) \\
& \times\left[\tilde{G}_{p q}\left(k_{x}, k_{y}\right)-\tilde{G}_{p q}^{\infty}\left(k_{x}, k_{y}\right)\right] \tilde{J}_{m n}^{q}\left(k_{x}, k_{y}\right) d k_{x} d k_{y} \\
& -\frac{1}{4 \pi^{2}} \int_{\infty}^{\infty} \int_{\infty}^{\infty} \tilde{J}_{m^{\prime} n^{\prime}}^{p^{*}}\left(k_{x}, k_{y}\right) \tilde{G}_{p q}^{\infty}\left(k_{x}, k_{y}\right) \tilde{J}_{m n}^{q}\left(k_{x}, k_{y}\right) d k_{x} d k_{y}
\end{aligned}
$$

( $p=x$ or $y$, and $q=x$ or $y$ ) where $Z_{m^{\prime} n^{\prime} m n}^{p q}$ represents the self and mutual interactions between the roof-top subdomain current basis functions $J_{m^{\prime} n^{\prime}}^{p}$ and $J_{m n}^{q}$. In Eq. (1), $\tilde{J}_{m^{\prime} n^{\prime}}^{p}$ is the Fourier transform of the $p$-directed basis function (i.e., $J_{m^{\prime} n^{\prime}}^{p}$ ), $\widetilde{J}_{m n}^{q^{*}}$ is the complex conjugate of the Fourier transform of the $q$-directed basis function, and finally $\tilde{G}_{p q}$ is the appropriate dyadic Green's function component in the spectral domain with $\tilde{G}_{p q}^{\infty}$ being its asymptotic value for large 
$\beta=\sqrt{k_{x}^{2}+k_{y}^{2}}$ values. The explicit expressions for $J_{m^{\prime} n^{\prime}}^{p}$, its Fourier transform, $\tilde{G}_{p q}$ and $\tilde{G}_{p q}^{\infty}$ are given in Ref. 6. In a similar fashion, the MoM excitation vector elements (for probe-fed structures) are expressed as

$$
\begin{array}{r}
V_{m^{\prime} n^{\prime}}^{q}=\frac{1}{4 \pi^{2}} \int_{\infty}^{\infty} \int_{\infty}^{\infty}\left[\tilde{G}_{z q}\left(k_{x}, k_{y}\right)-\tilde{G}_{z q}^{\infty}\left(k_{x}, k_{y}\right)\right] \tilde{J}_{m^{\prime} n^{\prime}}^{q}\left(k_{x}, k_{y}\right) \\
\times e^{j\left(k_{x} x^{p}+k_{y y} y^{p}\right)} d k_{x} d k_{y}+\frac{1}{4 \pi^{2}} \int_{\infty}^{\infty} \int_{\infty}^{\infty} \tilde{G}_{z q}^{\infty}\left(k_{x}, k_{y}\right) \tilde{J}_{m^{\prime} n^{\prime}}^{q}\left(k_{x}, k_{y}\right) \\
\times e^{j\left(k_{x} x^{p}+k_{y} y^{p}\right)} d k_{x} d k_{y}
\end{array}
$$

where $\left(x^{p}, y^{p}\right)$ is the coaxial probe attachment position on the patch surface and $\tilde{G}_{z q}$ is appropriate dyadic Green's function component in the spectral domain with $\tilde{G}_{z q}^{\infty}$ being its asymptotic value for large $\beta$ values. The explicit expressions for $\tilde{G}_{z q}$ and $\tilde{G}_{z q}^{\infty}$ are given in Refs. 1 and 7.

In the first terms of Eqs. (1) and (2), the infinite double integrals converge rapidly to zero. However, the second terms in Eqs. (1) and (2) (called as the asymptotic part of the impedance matrix element and the MoM excitation vector element) also contain the infinite double integrals which exhibit slowly convergent and highly oscillatory behavior. Therefore, in Refs. 5 and 6 an analytical technique has been derived for the fast and accurate evaluation of the asymptotic part of the impedance matrix elements, and then this technique has been applied to the MoM excitation vector elements in Ref. 7. Consequently, the infinite double integrals in the asymptotic part of Eqs. (1) and (2) are analytically transformed to $1 \mathrm{D}$ integrals given by

$$
\begin{gathered}
I_{m^{\prime} n^{\prime} m n}^{x^{a} m}=\frac{1}{\pi} \int_{-2 \Delta x}^{2 \Delta x} A\left(\chi-x_{s}\right) \mathfrak{\Im}_{a}(\chi) d \chi \\
I_{m^{\prime} n^{\prime} m n}^{x x^{b}}=\frac{1}{\pi} \int_{-2 \Delta x}^{2 \Delta x} A\left(\chi-x_{s}\right) \Im_{b}(\chi) d \chi \\
I_{m^{\prime} n^{\prime} m n}^{x y}=\frac{1}{\pi} \int_{-\frac{3 \Delta x}{2}+x_{s}}^{\frac{3 \Delta x}{2}+x_{s}} B(\chi) T\left(\chi-x_{s}\right) d \chi \\
C(\chi) \Gamma\left(\chi+x_{A}\right) d \chi \\
-\frac{1}{2 \pi} \int_{-x_{A}-\Delta x}^{-x_{A}+\Delta x} \int_{x_{A}-\Delta x}^{x_{A}+\Delta x} C(\chi) \Gamma\left(\chi-x_{A}\right) d \chi,
\end{gathered}
$$

where the closed-form expressions for $A\left(\chi-x_{s}\right), \Im_{a}(\chi)$, $\mathfrak{\Im}_{b}(\chi), B(\chi), T\left(\chi-x_{s}\right), C(\chi)$ and $\Gamma\left(\chi-x_{A}\right)$ are given in Refs. 6 and 7. Basically, $A\left(\chi-x_{s}\right)$ is in Eqs. (39), (40), and (41), $\mathfrak{\Im}_{a}(\chi)$ in Eq. (26), $\Im_{b}(\chi)$ in Eq. (27), $B(\chi)$ in Eq. (45), and $T\left(\chi-x_{s}\right)$ in Eq. (30) of Ref. 6, and $C(\chi)$ in Eq. (14) and $\Gamma\left(\chi-x_{A}\right)$ in Eq. (11) of Ref. 7, respectively. Similar expressions are formed for $I_{m^{\prime} n^{\prime} m n}^{y a}$, $I_{m^{\prime} n^{\prime} m n}^{y y^{b}}, I_{m^{\prime} n^{\prime} m n}^{y x}$, and $I_{m^{\prime} n^{\prime}}^{y}$ by interchanging $\Delta x \leftrightarrow \Delta y, x_{s} \leftrightarrow y_{s}$ and $x_{A}$ $\leftrightarrow y_{A}$, where $x_{s}$ and $y_{s}$ are the lateral separation between the basis and testing functions (i.e., $x_{s}=x_{m}-x_{m^{\prime}} ; y_{s}=y_{n}-y_{n^{\prime}}$ ), $x_{A}$ and $y_{A}$ are the separation between the basis function under analysis and the probe location (i.e., $x_{A}=x_{p}-x_{m^{\prime}} ; y_{A}=y_{p}-y_{n^{\prime}}$ ), and $\Delta x$ and
$\Delta y$ are related with the size of the roof-top functions as explained in Ref. 6 such that roof-top functions for the $\hat{x}$-directed current elements have dimensions $2 \Delta x$ and $\Delta y$ in the $\hat{x}$ and $\hat{y}$ directions, respectively, while the size of roof-top for the $\hat{y}$-directed current elements have dimensions $\Delta x$ and $2 \Delta y$ in the $\hat{x}$ and $\hat{y}$ directions, respectively.

In Refs. 5-7, the 1D integrals given in Eqs. (3)-(6) were computed numerically using the IMSL subroutines. During the computation of these integrals, if there is a singularity at the integration interval, then the IMSL routine DQDAGP was used, which can handle interior and endpoint singularities. If there is no singularity, the IMSL routine DQDAGS was used. Unfortunately, these routines are highly specialized and may not be available on all platforms. Besides, it is observed that using standard numerical integration techniques instead of these IMSL routines yields accuracy problems. Therefore, in this study, we provide closed-form expressions for these integrals. The key steps in arriving at these closed-form expressions are

i. The analytic evaluation of the following type integrals:

$$
\begin{gathered}
f_{i}\left(a, x_{1}, x_{2}\right)=\int_{x_{1}}^{x_{2}} x^{i} \sqrt{x^{2}+a^{2}} d x, \\
F_{i}\left(a, x_{1}, x_{2}, x_{s}\right)=\int_{x_{1}}^{x_{2}} x^{i} \sqrt{\left(x-x_{s}\right)^{2}+a^{2}} d x, \\
g_{i}\left(a, x_{1}, x_{2}\right)=\int_{x_{1}}^{x_{2}} x^{i} \ln \left(a+\sqrt{x^{2}+a^{2}}\right) d x, \\
G_{i}\left(a, x_{1}, x_{2}, x_{s}\right)=\int_{x_{1}}^{x_{2}} x^{i} \ln \left(a+\sqrt{\left(x-x_{s}\right)^{2}+a^{2}}\right) d x
\end{gathered}
$$

with $i=0,1,2,3$. It is important to notice that $F_{i}\left(a, x_{1}, x_{2}, x_{s}\right)$ and $G_{i}\left(a, x_{1}, x_{2}, x_{s}\right)$ are expressed in terms of $f_{i}\left(a, x_{1}, x_{2}\right)$ and $g_{i}\left(a, x_{1}\right.$, $\left.x_{2}\right)$, respectively.

ii. Recognizing that the closed-form expressions to the integrals given by Eqs. (3)-(6) can be obtained as a combination of Eqs. (7)-(10).

Consequently, the closed-form expressions for the $1 \mathrm{D}$ integrals given by Eqs. (3)-(6) are found as follows:

$$
\begin{aligned}
I_{m^{\prime} n^{\prime} m n}^{x x^{a}}=\frac{\pi}{768} \sum_{q=1}^{2} \sum_{p=1}^{3} & \sum_{i=0}^{3}\left\{c _ { i } ^ { s _ { 1 } } ( \Delta x , q ) \left[c_{g}(p) G_{i}\left(a_{p}^{x x}, \chi_{3 q-2}^{x x}, \chi_{3 q-1}^{x x}, x_{s}\right)\right.\right. \\
+ & \left.\left.c_{f}(p) F_{i}\left(a_{p}^{x x}, \chi_{3 q-2}^{x x}, \chi_{3 q-1}^{x x}, x_{s}\right)\right]\right\} \\
& +\frac{\pi}{768} \sum_{q=1}^{2} \sum_{p=1}^{3} \sum_{i=0}^{3}\left\{c_{i}^{s_{2}}(\Delta x, q)\right. \\
& \times\left[c_{g}(p) G_{i}\left(a_{p}^{x x}, \chi_{q+1}^{x x}, \chi_{q+2}^{x x}, x_{s}\right)\right. \\
& \left.\left.+c_{f}(p) F_{i}\left(a_{p}^{x x}, \chi_{q+1}^{x x}, \chi_{q+2}^{x x}, x_{s}\right)\right]\right\}
\end{aligned}
$$

$$
I_{m^{\prime} n^{\prime} m n}^{x x^{b}}=\frac{\pi}{16} \sum_{q=1}^{2} \sum_{p=1}^{3} \sum_{i=0}^{1}\left\{c _ { i } ^ { s _ { 3 } } ( \Delta x , q ) \left[c_{g}(p) G_{i}\left(a_{p}^{x x}, \chi_{3 q 2}^{x x}, \chi_{3 q-1}^{x x}, x_{s}\right)\right.\right.
$$




$$
\begin{gathered}
\left.\left.+c_{f}(p) F_{i}\left(a_{p}^{x x}, \chi_{3 q-2}^{x x}, \chi_{3 q-1}^{x x}, x_{s}\right)\right]\right\}+\frac{\pi}{16} \sum_{q=1}^{2} \sum_{p=1}^{3} \sum_{i=0}^{1}\left\{C_{i}^{s u}(\Delta x, q)\right. \\
\times\left[c_{g}(p) G_{i}\left(a_{p}^{x x}, \chi_{q+1}^{x x}, \chi_{q+2}^{x x}, x_{s}\right)\right. \\
\left.\left.+c_{f}(p) F_{i}\left(a_{p}^{x x}, \chi_{q+1}^{x x}, \chi_{q+2}^{x x}, x_{s}\right)\right]\right\}
\end{gathered}
$$

$$
\begin{aligned}
I_{m^{\prime} n^{\prime} m n}^{x y}= & \sum_{q=1}^{4} \sum_{p=1}^{3}\left\{d ^ { x y } ( q ) \left[c ^ { x y } ( 2 p - 1 ) \left(f_{0}\left(a_{q}^{x y}, \chi_{p}^{x y}, \chi_{p+1}^{x y}\right)\right.\right.\right. \\
& \left.-a_{q}^{x y} g_{0}\left(a_{q}^{x y}, \chi_{p}^{x y}, \chi_{p+1}^{x y}\right)\right)+c^{x y}(2 p)\left(f_{1}\left(a_{q}^{x y}, \chi_{p}^{x y}, \chi_{p+1}^{x y}\right)\right. \\
& \left.\left.-a_{q}^{x y} g_{1}\left(a_{q}^{x y}, \chi_{p}^{x y}, \chi_{p+1}^{x y}\right)\right)\right\}
\end{aligned}
$$

$$
\begin{aligned}
I_{m^{\prime} n^{\prime}}^{2 x}=g_{0}\left(a_{1}^{z x}, \chi_{1}^{z x}, \chi_{2}^{z x}\right)-g_{0}\left(a_{2}^{2 x}, \chi_{1}^{2 x}, \chi_{2}^{z x}\right)- & g_{0}\left(a_{1}^{z x}, \chi_{2}^{z x}, \chi_{3}^{z x}\right) \\
& +g_{0}\left(a_{2}^{z x}, \chi_{2}^{z x}, \chi_{3}^{z x}\right) .
\end{aligned}
$$

In Eqs. (11) and (12), the constants and the coefficients are given as follows:

$$
\begin{gathered}
c_{0}^{s_{1}}=8 \Delta x^{3} ; c_{1}^{s_{1}}=12 \Delta x^{2}(-1)^{q+1} ; c_{2}^{s_{1}}=6 \Delta x ; c_{3}^{s_{1}}=(-1)^{q+1}, \\
c_{0}^{s_{2}}=-4 \Delta x^{3} ; c_{1}^{s_{2}}=0 ; c_{2}^{s_{2}}=-6 \Delta x ; c_{3}^{s_{2}}=3(-1)^{q} \\
c_{0}^{s_{3}}=-\frac{\Delta x}{4} ; c_{1}^{s_{3}}=\frac{1}{8}(-1)^{q} ; c_{0}^{s_{4}}=\frac{\Delta x}{4} ; c_{1}^{s_{4}}=\frac{3}{8}(-1)^{q+1} \\
\chi_{1}^{x x}=-2 \Delta x ; \chi_{2}^{x x}=-\Delta x ; \chi_{3}^{x x}=0 ; \chi_{4}^{x x}=\Delta x ; \chi_{5}^{x x}=2 \Delta x \\
a_{1}^{x x}=y_{s}+\Delta y ; a_{2}^{x x}=y_{s}-\Delta y ; a_{3}^{x x}=y_{s} \\
c_{g}(1)=y_{s}+\Delta y ; c_{g}(2)=y_{s}-\Delta y ; c_{g}(3)=-2 y_{s} \\
c_{f}(1)=-1 ; c_{f}(2)=-1 ; c_{f}(3)=2
\end{gathered}
$$

Similarly, in Eqs. (13) and (14), the constants and the coefficients are as follows:

$$
\begin{aligned}
& \chi_{1}^{x y}=-1.5 \Delta x+x_{s} ; \chi_{2}^{x y}=-0.5 \Delta x+x_{s} ; \chi_{3}^{x y} \\
& =0.5 \Delta x+x_{s} ; \chi_{4}^{x y}=1.5 \Delta x+x_{s}, \\
& a_{1}^{x y}=-1.5 \Delta y+y_{s} ; a_{2}^{x y}=-0.5 \Delta y+y_{s} ; a_{3}^{x y} \\
& =0.5 \Delta y+y_{s} ; a_{4}^{x y}=1.5 \Delta y+y_{s}, \\
& c^{x y}(1)=-\frac{\pi}{8}\left(1.5 \Delta x-x_{s}\right) ; c^{x y}(2)=-\frac{\pi}{8} ; c^{x y}(3)=-\frac{\pi}{4} x_{s} ; \\
& c^{x y}(4)=\frac{\pi}{4} ; c^{x y}(5)=\frac{\pi}{8}\left(1.5 \Delta x+x_{s}\right) ; c^{x y}(6)=-\frac{\pi}{8}, \\
& d^{x y}(1)=-\frac{1}{16} ; d^{x y}(2)=\frac{3}{16} ; d^{x y}(3)=-\frac{3}{16} ; d^{x y}(4)=\frac{1}{16}, \\
& \chi_{1}^{z x}=x_{p}-\Delta x ; \chi_{2}^{z x}=x_{p} ; \chi_{3}^{z x}=x_{p}+\Delta x, \\
& a_{1}^{z x}=y_{p}+\frac{\Delta y}{2} ;{ }_{2}^{z x}=y_{p}-\frac{\Delta y}{2} .
\end{aligned}
$$

Finally, the analytical expressions to the results of the integrals defined in Eqs. (7)-(10), which are the main building blocks of Eqs. (11)-(14), are given by

$$
f_{0}\left(a, x_{1}, x_{2}\right)=\frac{x_{2}}{2} \sqrt{x_{2}^{2}+a^{2}}-\frac{x_{1}}{2} \sqrt{x_{1}^{2}+a^{2}}+\frac{a^{2}}{2} \ln \left(\frac{x_{2}+\sqrt{x_{2}^{2}+a^{2}}}{x_{1}+\sqrt{x_{1}^{2}+a^{2}}}\right),
$$

$$
\begin{gathered}
f_{1}\left(a, x_{1}, x_{2}\right)=\left(x_{2}^{2}+a^{2}\right)^{3 / 2}-\left(x_{1}^{2}+a^{2}\right)^{3 / 2}, \\
f_{2}\left(a, x_{1}, x_{2}\right)=\frac{\left[x_{2}\left(x_{2}^{2}+a^{2}\right)^{3 / 2}-x_{1}\left(x_{1}^{2}+a^{2}\right)^{3 / 2}\right]}{4} \\
-\frac{a^{2}}{8}\left[x_{2}\left(x_{2}^{2}+a^{2}\right)^{1 / 2}-x_{1}\left(x_{1}^{2}+a^{2}\right)^{1 / 2}\right]-\frac{a^{4}}{8} \ln \left(\frac{x_{2}+\sqrt{a^{2}+x_{2}^{2}}}{x_{1}+\sqrt{a^{2}+x_{1}^{2}}}\right),
\end{gathered}
$$

$f_{3}\left(a, x_{1}, x_{2}\right)=\frac{1}{15}\left(x_{2}^{2}+a^{2}\right)^{3 / 2}\left(3 x_{2}^{2}-2 a^{2}\right)$

$$
-\frac{1}{15}\left(x_{1}^{2}+a^{2}\right)^{3 / 2}\left(3 x_{1}^{2}-2 a^{2}\right),
$$

$$
F_{0}\left(a, x_{1}, x_{2}, x_{s}\right)=f_{0}\left(a, x_{1}-x_{s}, x_{2}-x_{s}\right),
$$

$F_{1}\left(a, x_{1}, x_{2}, x_{s}\right)=f_{1}\left(a, x_{1}-x_{s}, x_{2}-x_{s}\right)$

$$
+x_{s} f_{0}\left(a, x_{1}-x_{s}, x_{2}-x_{s}\right),
$$

$F_{2}\left(a, x_{1}, x_{2}, x_{s}\right)=f_{2}\left(a, x_{1}-x_{s}, x_{2}-x_{s}\right)+2 x_{s} f_{1}\left(a, x_{1}-x_{s}, x_{2}\right.$

$$
\left.-x_{s}\right)+x_{s}^{2} f_{0}\left(a, x_{1}-x_{s}, x_{2}-x_{s}\right)
$$

$F_{3}\left(a, x_{1}, x_{2}, x_{s}\right)=f_{3}\left(a, x_{1}-x_{s}, x_{2}-x_{s}\right)+3 x_{s} f_{2}\left(a, x_{1}-x_{s}, x_{2}\right.$

$$
\left.-x_{s}\right)+3 x_{s}^{2} f_{1}\left(a, x_{1}-x_{s}, x_{2}-x_{s}\right)+x_{s}^{3} f_{0}\left(a, x_{1}-x_{s}, x_{2}-x_{s}\right),
$$

$g_{0}\left(a, x_{1}, x_{2}\right)=2_{2} \ln \left(a+\sqrt{x_{2}^{2}+a^{2}}\right)-x_{1} \ln \left(a+\sqrt{x_{1}^{2}+a^{2}}\right)$

$$
-\left(x_{2}-x_{1}\right)+a \ln \left(\frac{x_{2}+\sqrt{a^{2}+x_{2}^{2}}}{x_{1}+\sqrt{a^{2}+x_{1}^{2}}}\right)
$$

$g_{1}\left(a, x_{1}, x_{2}\right)=\frac{x_{2}^{2}}{2} \ln \left(a+\sqrt{x_{2}^{2}+a^{2}}\right)-\frac{x_{1}^{2}}{2} \ln \left(a+\sqrt{x_{1}^{2}+a^{2}}\right)$

$$
+\frac{a}{2}\left(\sqrt{x_{2}^{2}+a^{2}}-\sqrt{x_{1}^{2}+a^{2}}\right)-\frac{1}{4}\left(x_{2}^{2}-x_{1}^{2}\right)
$$

$$
\begin{array}{r}
g_{2}\left(a, x_{1}, x_{2}\right)=\frac{x_{2}^{3}}{3} \ln \left(a+\sqrt{x_{2}^{2}+a^{2}}\right)-\frac{x_{1}^{3}}{3} \ln \left(a+\sqrt{x_{1}^{2}+a^{2}}\right) \\
-\frac{\left(x_{2}^{3}-x_{1}^{3}\right)}{9}+\frac{a}{6}\left(x_{2} \sqrt{x_{2}^{2}+a^{2}}-x_{1} \sqrt{x_{1}^{2}+a^{2}}\right) \\
-\frac{a^{3}}{6} \ln \left(\frac{x_{2}+\sqrt{x_{2}^{2}+a^{2}}}{x_{2}+\sqrt{x_{1}^{2}+a^{2}}}\right),
\end{array}
$$

$$
\begin{gathered}
g_{3}\left(a, x_{1}, x_{2}\right)=\frac{x_{2}^{4}}{4} \ln \left(a+\sqrt{x_{2}^{2}+a^{2}}\right)-\frac{x_{1}^{4}}{4} \ln \left(a+\sqrt{x_{1}^{2}+a^{2}}\right) \\
-\frac{\left(x_{2}^{4}-x_{1}^{4}\right.}{16}+\frac{a\left(x_{2}^{2}-2 a^{2}\right.}{12} \sqrt{x_{2}^{2}+a^{2}}-\frac{a\left(x_{1}^{2}-2 a^{2}\right)}{12} \sqrt{x_{1}^{2}+a^{2}},
\end{gathered}
$$




$$
\begin{gathered}
G_{0}\left(a, x_{1}, x_{2}, x_{s}\right)=g_{0}\left(a, x_{1}-x_{2}-x_{s}\right), \\
G_{1}\left(a, x_{1}, x_{2}, x_{s}\right)=g_{1}\left(a, x_{1}-x_{s}, x_{2}-x_{s}\right) \\
+x_{s} g_{0}\left(a, x_{1}-x_{s}, x_{2}-x_{s}\right), \\
G_{2}\left(a, x_{1}, x_{2}, x_{s}\right)=g_{2}\left(a, x_{1}-x_{s}, x_{2}-x_{s}\right)+2 x_{s} g_{1}\left(a, x_{1}-x_{s}, x_{2}\right. \\
\left.-x_{s}\right) x_{s}^{2} g_{0}\left(a, x_{1}-x_{s}, x_{2}-x_{s}\right),
\end{gathered}
$$

\section{NUMERICAL RESULTS AND DISCUSSION}

To assess the accuracy of the closed-form expressions presented in Eqs. (11)-(14) with the related parameters given by Eqs. (15)-(43), several numerical results in the form of mutual impedance between two expansion functions and the input impedance of several probe-fed microstrip patch antennas are obtained and compared with the simulation and measurement results available in the literature.

The first numerical example is the duplication of Figure 2 in Ref. 6, where the finite 1D integrals are compared with the double infinite integrals using $\Delta x=\Delta y=1$ and $y_{s}=2 \Delta y$ for $0 \leq x_{s} \leq$ 10 for Eqs. (3) and (4), and using $\Delta x=\Delta y=1$ and $y_{s}=3 / 2 \Delta y$ for $0 \leq x_{s} \leq 10$ for Eq. (5). We also evaluated the same integrals, Eqs. (3)-(5), using the closed-form expressions. As depicted in Figure 1; excellent agreement is obtained.

As a second example, the mutual interaction between two $\hat{x}$-directed current modes, which are defined to be roof-top functions [Eq. (8) in Ref. 6], are evaluated along the H-plane (i.e., along the $y$-axis). These current modes are on a grounded dielectric slab with a thickness, $h=0.057 \lambda_{0}$ ( $\lambda_{0}$ is the free-space wavelength) and $\varepsilon_{\mathrm{r}}=$ 2.33, and the size of each current mode is selected to be $\Delta x=0.05 \lambda_{0}$ and $\Delta y=0.025 \lambda_{0}$. Since IMSL routines are highly specialized and are not available on our platforms, we used the standard Gaussian quadrature algorithm in the following way: For the integration limits from $-2 \Delta x$ to $2 \Delta x$, we divided the integration interval to subintervals with the subinterval length being $\Delta x / 8$. In each subinterval we used an 8-point Gaussian quadrature algorithm. As seen in Figure 2, we have an excellent agreement both in magnitude and phase, except for relatively large separations, where the finite 1D integration method yields some numerical problems. As a result, we believe this result illustrates the importance of the closed-form expressions that we provide for the $1 \mathrm{D}$ integrals.

The last two numerical examples, shown in Figures 3 and 4, provide the Smith Chart plots of the input impedance of two probe-fed microstrip antennas, where the closed-form expressions for both the impedance matrix and the excitation vector are used. Results are also compared with the previously published results as well as the results of a software package ENSEMBLE [8]. Figure 3 is given for a rectangular microstrip patch antenna on a grounded dielectric slab with $\varepsilon_{\mathrm{r}}=10.2$ and thickness $h=0.127 \mathrm{~cm}$. The length of the patch $L$ is $2 \mathrm{~cm}$, the width of the patch $W$ is $3 \mathrm{~cm}$, and the feed is located $1 \mathrm{~cm}$ from the long edge (i.e., from the $W$ edge) and $0.65 \mathrm{~cm}$ from the short edge (i.e., from the $L$ edge) as explained in Ref. 9. The frequency is varied from 2.2 to $2.4 \mathrm{GHz}$, and 9 roof-top basis functions are used along the width of the patch. As seen in Figure 3, very good agreement is obtained with both the measured results given in Ref. 9 and the results obtained from the ENSEMBLE software [8].

In a similar fashion Figure 4 is given for $W=39.52 \mathrm{~mm}$ by $L=$ $49.91 \mathrm{~mm}$ rectangular antenna with a coaxial feed located at $W / 2$
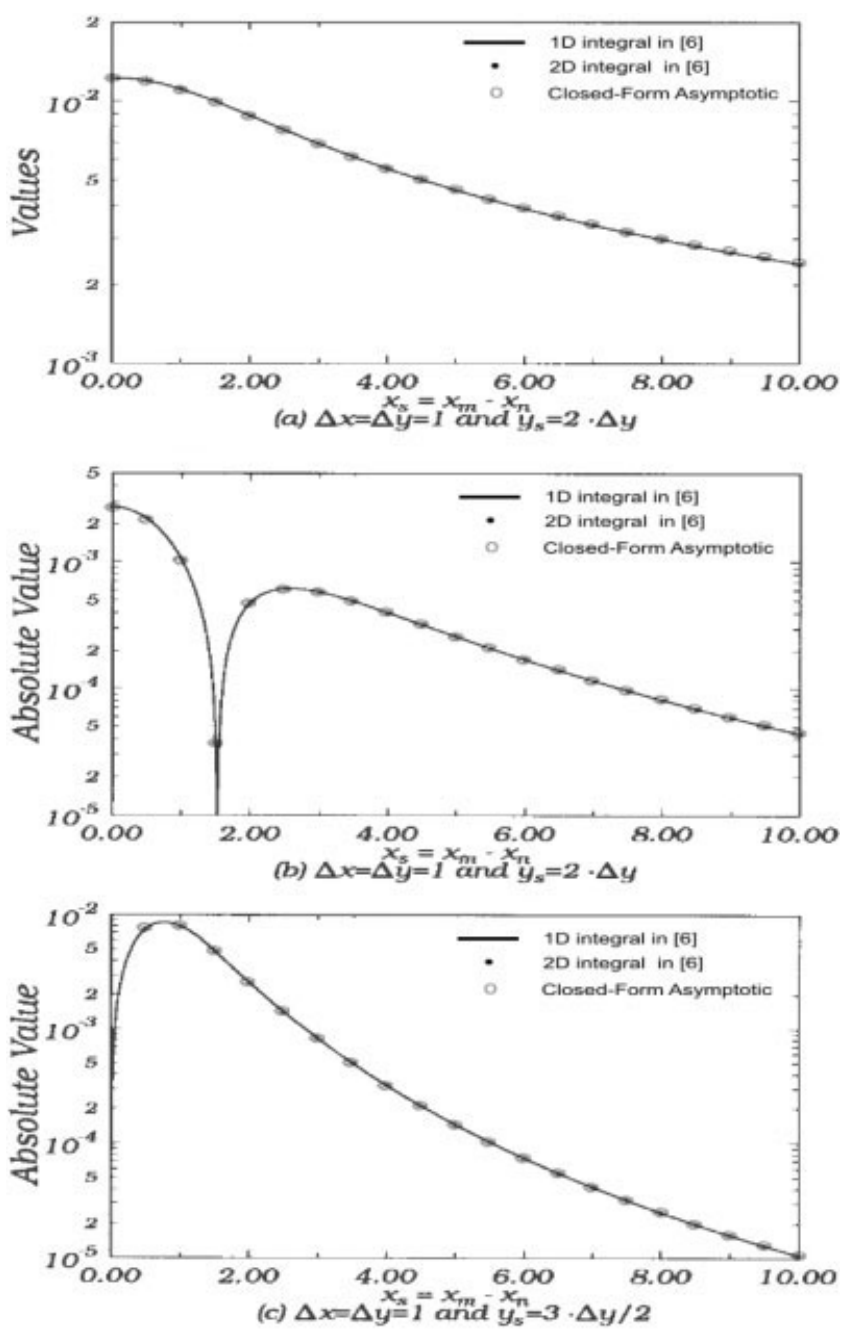

Figure 1 Comparison among the infinite 2D integral, the finite 1D integral, and the closed-form expressions

from the long side (i.e., from the $L$ edge) and $15.36 \mathrm{~mm}$ from the short side (i.e., from the $W$ edge) as depicted in Ref. 10. The antenna is located on a grounded dielectric slab with $\varepsilon_{\mathrm{r}}=2.484$ and $h=6.3 \mathrm{~mm}$. The frequency is varied from 1.72 to $2.10 \mathrm{GHz}$, and five roof-top basis functions are used along the length of the patch. Similar to the previous case, very good agreement is obtained with both the measured and the simulated results given in Ref. 10 as well as the results obtained from the ENSEMBLE software [8]. Note that to account the self inductance of the probe we added $j X_{\mathrm{pr}}$ to the input impedance given by

$$
X_{\mathrm{pr}}=-\frac{\eta k h}{2 \pi}\left[\ln \left(\frac{k d}{4}\right)+0.577\right]
$$

where $\eta$ is the intrinsic impedance of the dielectric medium, $k$ is the wave number of the dielectric medium, $d$ is the diameter of the feed probe, and $h$ is the thickness of the substrate [11].

\section{CONCLUSIONS}

Closed-form expressions are derived for the asymptotic parts of both the impedance matrix and the excitation vector of probe-fed printed geometries when spectral domain MoM solution is employed. Previously, these asymptotic parts have been expressed as the finite $1 \mathrm{D}$ integrals that are computed using the IMSL subrou- 

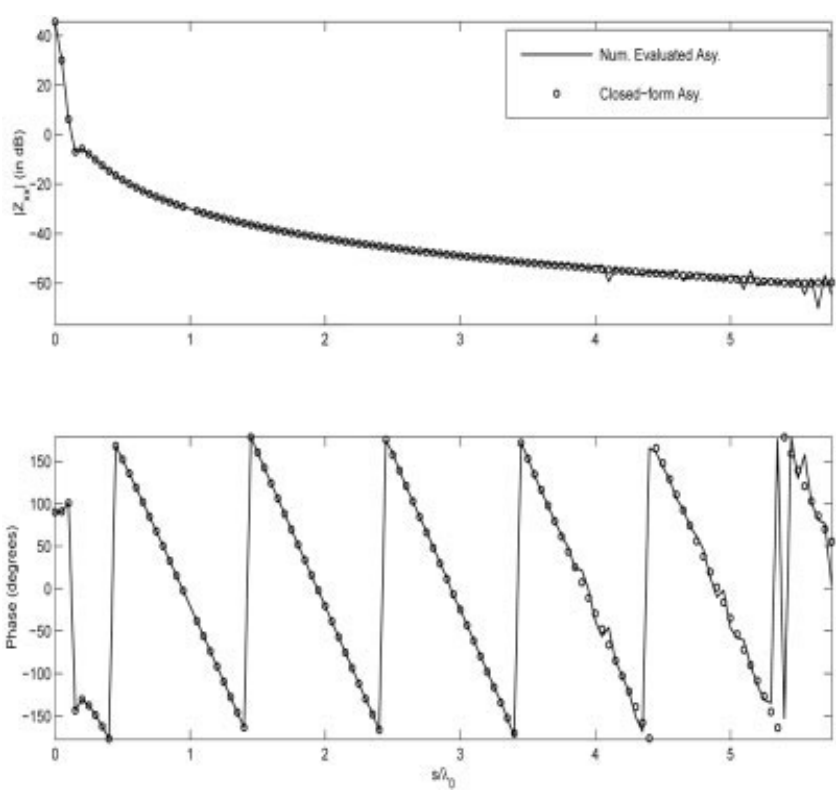

Figure 2 Magnitude and phase variations of the mutual impedance $Z_{12}^{x x}$ between two identical $\hat{x}$-directed current modes versus separation $\left(s / \lambda_{0}\right)$ on a $h=0.057 \lambda_{0}$ thick grounded dielectric slab with $\varepsilon_{\mathrm{r}}=2.33$

tines DQDAGP and DQDAGS, which are highly specialized and may not be available on all platforms. Besides, it has been observed that using standard numerical integration techniques instead of these IMSL routines may yield accuracy problems. Consequently, implementation of these closed-form expressions to our existing spectral domain MoM codes eliminates the need for these specialized subroutines, and results in a further reduction in the CPU time and a further improvement in the accuracy for the evaluation of the MoM matrix and the excitation vector entries as illustrated in the numerical results in the form of mutual coupling

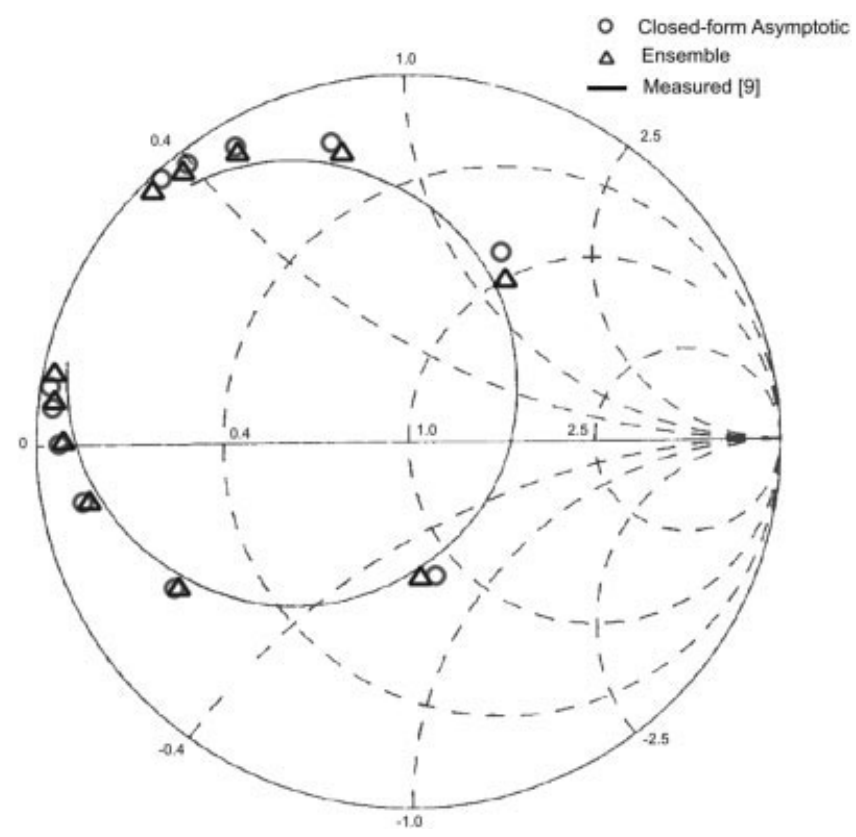

Figure 3 Input impedance data of a probe-fed $L=2 \mathrm{~cm}$ by $W=3 \mathrm{~cm}$ rectangular antenna on a $h=0.127 \mathrm{~cm}$ thick grounded dielectric slab with $\varepsilon_{\mathrm{r}}=10.2$. Frequency $=2.2-2.4 \mathrm{GHz}$

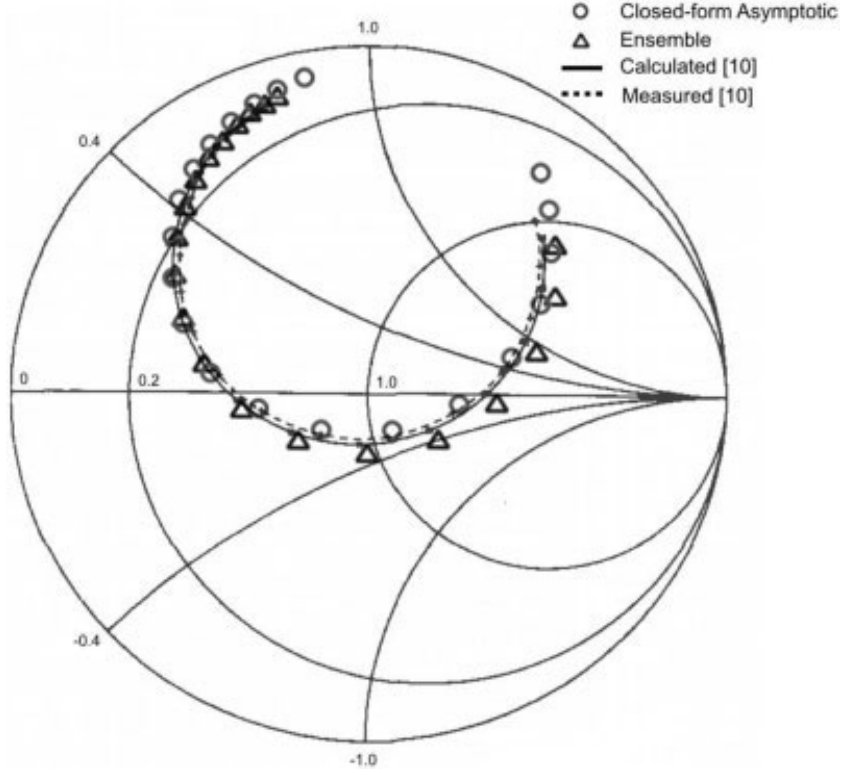

Figure 4 Input impedance data of a probe-fed $L=49.91 \mathrm{~mm}$ by $W=$ $39.52 \mathrm{~mm}$ rectangular antenna on a $h=6.3 \mathrm{~mm}$ thick grounded dielectric slab with $\varepsilon_{\mathrm{r}}=2.484$. Frequency $=1.72-2.10 \mathrm{GHz}$

between two current modes and the input impedance of various probe-fed microstrip antennas.

\section{ACKNOWLEDGMENTS}

This work was supported in part by the Turkish Scientific and Technological Research Agency (TÜBÍTAK) under grant no EEEAG-104E044.

\section{REFERENCES}

1. D.M. Pozar, Input impedance and mutual coupling of rectangular microstrip antennas, IEEE Trans Antennas Propagat 30 (1982), 1191-1196.

2. D.M. Pozar, Improved computational efficiency for the moment method solution of printed dipoles and patches, Electromagnetics 3 (1983), 299-309.

3. T. Vaupel and V. Hansen, Integral equation analysis of complex (M)MIC-structures with optimized system matrix decomposition and novel quadrature techniques, Adv Radio Sci 2 (2004), 101-105.

4. T. Vaupel, T.F. Eibert, and V. Hansen, Spectral domain analysis of large (M)MIC-structures using novel quadrature methods, Int J Num Model: Electron Networks Devices Fields 18 (2005), 23-38.

5. S.-O. Park and C.A. Balanis, Analytical technique to evaluate the asymptotic part of the impedance matrix of Sommerfeld-type integrals, IEEE Trans Antennas Propagat 45 (1997), 798-805.

6. S.-O. Park, C.A. Balanis, and C.R. Birtcher, Analytical evaluation of the asymptotic impedance matrix of a grounded dielectric slab with roof-top functions, IEEE Trans Antennas Propagat 46 (1998), 251-259.

7. F. Bilotti, A. Alu, F. Urbani, and L. Vegni, Asymptotic evaluation of the MOM excitation vector for probe-fed microstrip antennas, J Electromagn Waves Appl 19 (2005), 1639-1654.

8. Ansoft Corporation, ENSEMBLE Version 8.0. www.ansoft.com, Ansoft Corporation, Pittsburgh, PA.

9. D.H. Schaubert, D.M. Pozar, and A. Adrian, Effect of microstrip antenna substrate thickness and permittivity: comparison of theories with experiment. IEEE Trans Antennas Propagat 37 (1989), 677-682.

10. R.C. Hall and J.R. Mosig, Rigorous feed model for coaxially fed microstrip antenna, Electron Lett 26 (1990), 64-66.

11. C.A. Balanis, Antenna theory analysis and design, Wiley, New York, 1997.

(C) 2007 Wiley Periodicals, Inc. 